\title{
BMJ Open Effectiveness and cost-effectiveness of the Assessment of Burden of Chronic Conditions (ABCC) tool in patients with COPD, asthma, diabetes mellitus type 2 and heart failure: protocol for a pragmatic clustered quasi- experimental study
}

\author{
Esther A. Boudewijns (1) , ${ }^{1}$ Danny Claessens, ${ }^{1}$ Manuela Joore, ${ }^{2}$ \\ Lotte C.E.M. Keijsers, ${ }^{1}$ Onno C.P. van Schayck, ${ }^{1}$ Bjorn Winkens, ${ }^{3}$ \\ Annerika H.M. Gidding-Slok ${ }^{1}$
}

To cite: Boudewijns $E A$, Claessens D, Joore M, et al. Effectiveness and costeffectiveness of the Assessment of Burden of Chronic Conditions (ABCC) tool in patients with COPD, asthma, diabetes mellitus type 2 and heart failure: protocol for a pragmatic clustered quasiexperimental study. BMJ Open 2020;10:e037693. doi:10.1136/ bmjopen-2020-037693

- Prepublication history and additional materials for this paper are available online. To view these files, please visit the journal online (http://dx.doi. org/10.1136/bmjopen-2020037693).

Received 12 February 2020 Revised 24 August 2020 Accepted 25 September 2020

\section{Check for updates}

(c) Author(s) (or their employer(s)) 2020. Re-use permitted under CC BY-NC. No commercial re-use. See rights and permissions. Published by BMJ.

For numbered affiliations see end of article.

Correspondence to Esther A. Boudewijns; esther.boudewijns@ maastrichtuniversity.nl

\section{ABSTRACT}

Introduction The number of people that have one or multiple condition(s) with a chronic course is rising, which consequently challenges healthcare systems. Healthcare geared to long-term care should focus on patient-centredness, shared decision making and selfmanagement. The Assessment of Burden of Chronic Conditions $(A B C C)$ tool was developed to integrate these elements in daily healthcare practice. The ABCC tool assesses and visualises burden of disease(s), helps to make shared decisions and stimulates self-management. The present paper documents a protocol for a quasiexperimental study investigating the effectiveness and cost-effectiveness of the ABCC tool for people with chronic obstructive pulmonary disease, asthma, type 2 diabetes mellitus and/or heart failure.

Methods and analysis The study has a pragmatic clustered quasi-experimental design and will be conducted in the Netherlands. The intervention will be allocated at the level of general practice. The intervention group (18 general practices, 180 patients) will use the $A B C C$ tool during regular consultations; the control group (18 general practices, 180 patients) will maintain usual care. Outcomes include change in quality of care (Patient Assessment of Chronic Illness Care), quality of life (EuroQol-5D-5L), capability well-being (ICEpop CAPability measure for Adults), patients' activation (Patient Activation Measure) and costs. Follow-up time will be 18 months. Outcomes will be analysed using linear mixed models.

Ethics and dissemination Ethical approval was obtained from the Medical Ethics Committee ZuyderlandZuyd Heerlen, the Netherlands (METCZ20180131). Results will be published in peer-reviewed journals and will be presented at national and international conferences.

Trial registration number ClinicalTrials.gov Registry (NCT04127383).
Strengths and limitations of this study

The study is a large quasi-experimental trial and will provide important evidence on the effectiveness of a conversation and monitoring tool that promotes patient-centred care for people with one or multiple chronic conditions on perceived quality of care.

- The secondary outcomes of this study will offer the opportunity to also assess the effects on quality of life, capability well-being, patients' activation and cost-effectiveness of the Assessment of Burden of Chronic Conditions tool.

- The pragmatic approach will reflect the effectiveness in real-world implementation.

- A limitation of the study is a lack of randomisation, as this increases the risk of confounding due to an unequal distribution of prognostic factors.

\section{INTRODUCTION}

Amid concerns about providing high-quality care to increasing numbers of people with one or multiple chronic condition(s), soaring costs, and high expectations among patients and the public, reforms towards healthcare systems that are geared to provide long-term care are of growing importance. ${ }^{1-3}$ Consensus has been reached that these reforms should contain elements of patient-centred care and the Chronic Care Model. ${ }^{45}$ These include a regular assessment of the patient's problems; focus on quality of life, function and disease control; tailored treatment recommendations; promotion of self-management; shared decisions with the patient and an individualised care plan. ${ }^{1}$ 
Although a patient-centred approach is widely advocated, its implementation in everyday practice is limited. ${ }^{6}$ This might be due to a lack of practical support to encourage this way of working. ${ }^{3}$ Concerning chronic obstructive pulmonary disease (COPD), the Assessment of Burden of COPD (ABC) tool was therefore developed in 2014 to assess and visualise the burden of disease, to integrate this in a conversation based on the principles of shared decision making and to stimulate self-management. ${ }^{7}$ The $\mathrm{ABC}$ tool was shown to be valid, reliable and effective in improving quality of life and perceived quality of care. ${ }^{89}$ In general, patients and healthcare providers respond positively to the tool. ${ }^{10}$ Because burden of disease is not unique to COPD and because of the increasing number of people with multimorbidity, the $\mathrm{ABC}$ tool was reformed into the Assessment of Burden of Chronic Conditions (ABCC) tool for multiple chronic conditions in 2019. The ABCC tool is developed as a modular tool, in which a generic module will be combined with one or multiple disease-specific module(s) for COPD, asthma, type 2 diabetes mellitus and/or heart failure. The development of the tool has been described elsewhere. ${ }^{11}$ The current study aims to assess the effectiveness and cost-effectiveness of the ABCC tool in primary care. The primary research question is: What is the effect of the ABCC tool in patients with COPD, asthma, type 2 diabetes or heart failure (or a combination of these) on perceived quality of care, as measured by the Patient Assessment of Chronic Illness Care (PACIC), ${ }^{12} 13$ and compared with usual care after 18 months?

\section{METHODS AND ANALYSIS}

\section{Study design}

The study is designed to evaluate the effectiveness and cost-effectiveness of the ABCC tool in primary care in several regions throughout the Netherlands. The intervention group will use the ABCC tool and the control group will receive usual care. The ABCC tool is a sequel to the $\mathrm{ABC}$ tool, and the latter is currently being implemented in Dutch healthcare. Due to this fact, many healthcare providers are already using the $\mathrm{ABC}$ tool, and can therefore not participate in the control group of this study. Consequently, the number of healthcare providers that can participate in a randomised study is limited. Therefore, the study has a pragmatic clustered quasiexperimental design with an intervention group and a control group. To avoid contamination bias (ie, patients from the control group receiving the intervention because they are treated by the same healthcare provider or vice versa), the intervention will be allocated at the level of general practice. The study will have a follow-up period of 18 months.

\section{Study population, recruitment and allocation}

The researchers will recruit general practices. Within a general practice, one or more healthcare provider(s) (ie, general practitioners and practice nurses) can participate. Recruitment to the intervention or control group is based on whether the general practice has access to the ABCC tool. In the Netherlands, there are different systems for managing electronic medical records. These include systems for practice nurses and general practitioners: the Integrated Care Information System and the General Practice Information System, respectively. During the study, the ABCC tool will be available in one of the nine General Practice Information Systems, and in one Integrated Care Information System. General practices that have access to the ABCC tool will be allocated to the intervention group, and general practices that do not have access to the ABCC tool will be allocated to the control group.

The healthcare providers will subsequently recruit patients (figure 1). The reasons for this is that the healthcare provider and the patient together will either use the ABCC tool (intervention group) or give and receive usual care (control group). If a patient wants to participate and is eligible, the healthcare provider registers the patient on a website. The outcomes of the study are measured on a patient's level.

Because of the pragmatic design of the study, only a few eligibility criteria have been set. Healthcare providers will be recruited without specific criteria or prerequisites but will be excluded from the control group if they have

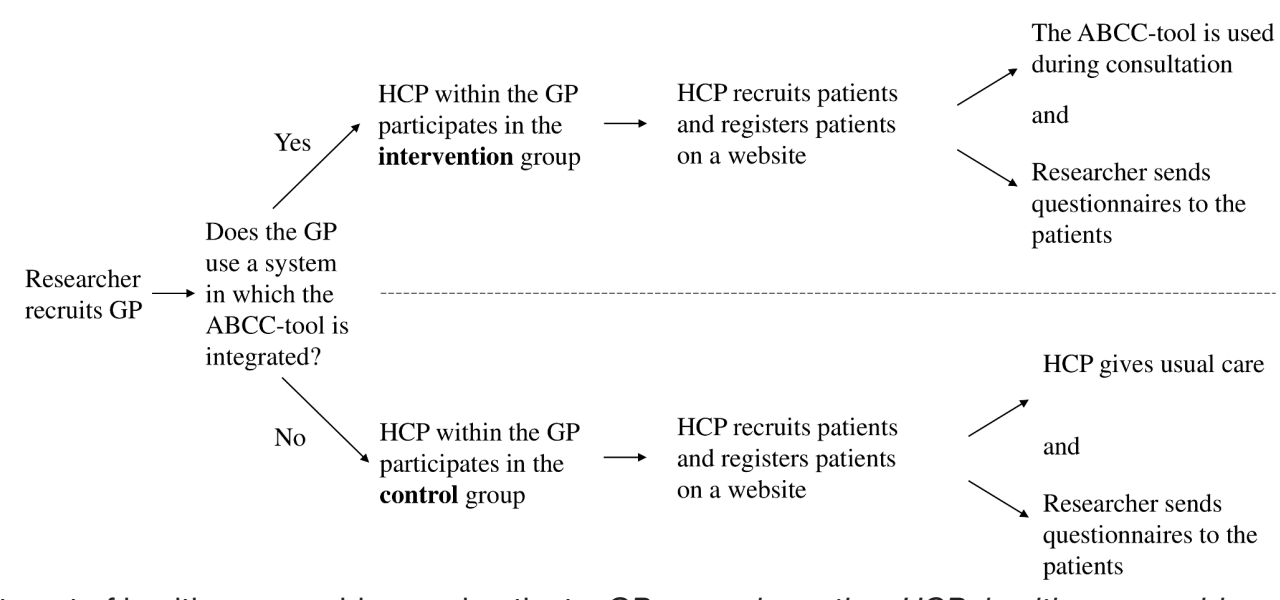

Figure 1 Recruitment of healthcare providers and patients. GP:general practice; HCP: healthcare provider 
already used the $\mathrm{ABC}$ tool. Concerning the intervention group, healthcare providers are allowed to have used the ABC tool. Patients are eligible if they have the diagnosis of COPD, asthma, type 2 diabetes and/or heart failure, are aged 18 or older, and can understand and read the Dutch language. Because the ABCC tool is a monitoring instrument (and not a diagnostic instrument), both new and existing patients can make use of the ABCC tool. To ensure that participants are stable at baseline, patients with asthma or COPD will not be eligible if they have used prednisone due to an exacerbation within the 6-week period prior to the commencement of the study, and patients with type 2 diabetes or heart failure will not be eligible if they were hospitalised within the 6-week period prior to the commencement of the study. Patients who have already used the ABC tool will also be excluded.

\section{Intervention}

Healthcare providers in the intervention group will be instructed to use the ABCC tool during usual consultation with their patients. ${ }^{11}$ The tool consists of a questionnaire, a balloon chart that is based on the outcomes of the questionnaire and treatment advice for each balloon. The questionnaire measures the experienced burden of the chronic condition(s) and several risk factors. It consists of a generic module, with items that are relevant for everyone with one of the aforementioned chronic conditions, as well as disease-specific modules. The generic questionnaire will be combined with any amount of disease-specific questionnaires (presently COPD, asthma, type 2 diabetes and heart failure), to form a single personalised scale and balloon chart for each individual patient. Patients will complete the questionnaire prior to the consultation with their healthcare provider. The balloon chart will be shown during the consultation and can be discussed by the healthcare provider and the patient (figure 2). A balloon represents a domain, and the colour and corresponding height indicate a patient's score on that domain. A red balloon indicates a low score, an orange balloon indicates a moderate score and a green balloon indicates a high score. The height and colour of the balloon is based on cut-off points reflecting the severity of the burden due to that specific domain, as described elsewhere. ${ }^{711}$ Displaying scores of the previous visit using grey balloons enables to monitor and visualise changes since the last visit. If a patient and a healthcare provider select a balloon and click on it, treatment advice will pop-up. This can support shared decision making between a patient and a healthcare provider. Based on the conversation following the treatment advice, personal care plans can be determined and, where applicable, treatment advice can be operationalised on the basis of shared decision making. ${ }^{14}$ Healthcare providers in the intervention group will receive a short instructional film about the ABCC tool before the start of the study as well as a poster that shortly displays the steps of the ABCC tool.

Healthcare providers in the control group will be instructed to provide usual care. This care is directed by national guidelines. Primary care will be given in accordance with the guidelines of the Dutch College of General Practitioners. ${ }^{15-18}$

Because the ABCC tool will be used in primary care, patients in the intervention and control group receive no instructions regarding the frequency of their visits. The frequency of visits depends on several factors, which are described in the guidelines of the Dutch College of General Practitioners. ${ }^{15-18}$ In general, patients with type 2 diabetes or heart failure visit their healthcare provider four times a year; patients with COPD with mild burden of disease or patients with well-controlled asthma visit their healthcare provider once a year. Therefore, it is expected that patients with COPD or asthma use the ABCC tool once or twice during the study, and patients with type 2 diabetes or heart failure will use the ABCC tool about six times during the study.

\section{Measurements}

Outcomes will be measured at a patient's level using questionnaires. The questionnaire within the ABCC tool, as described above, is part of the intervention and not

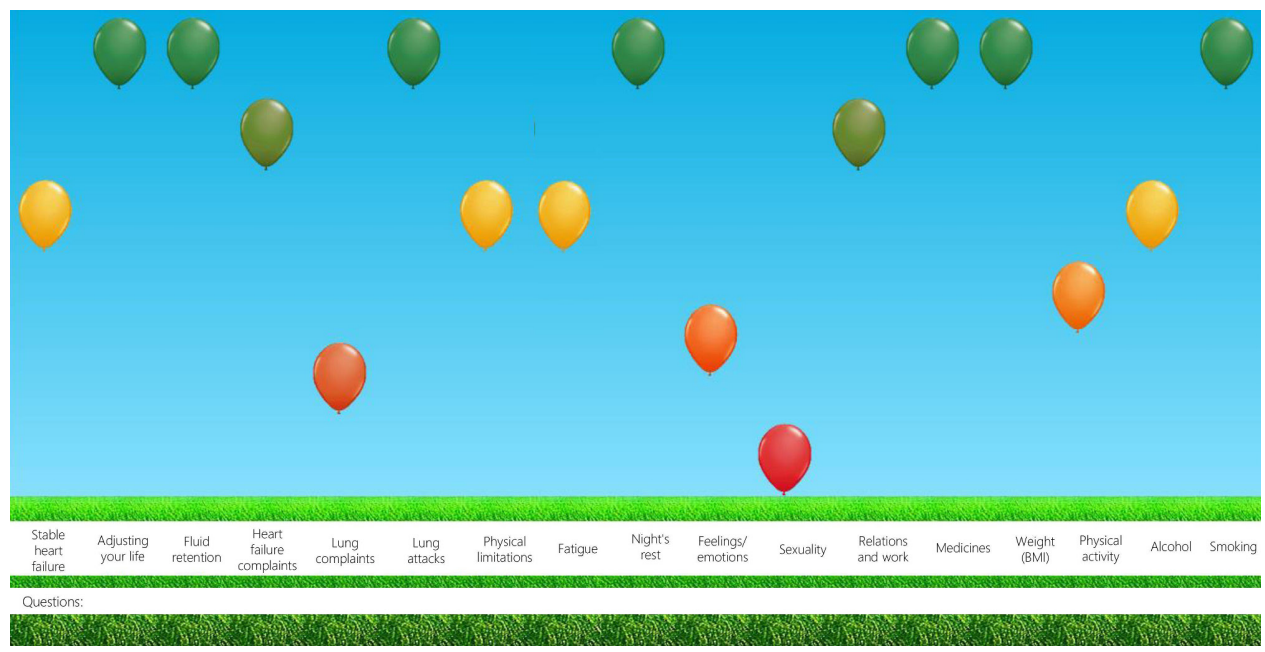

Figure 2 Visualisation of the integrated health status of a person with COPD and heart failure. 
Table 1 Overview of measurements of patient

Time (months)

\begin{tabular}{llllllll} 
& \multicolumn{1}{l}{ Time (months) } \\
\cline { 2 - 7 } & $\mathbf{0}$ & $\mathbf{3}$ & $\mathbf{6}$ & $\mathbf{9}$ & $\mathbf{1 2}$ & $\mathbf{1 5}$ & $\mathbf{1 8}$ \\
\hline Demographics & $\mathrm{x}$ & & & & & & \\
PACIC & $\mathrm{x}$ & & $\mathrm{x}$ & & $\mathrm{x}$ & & $\mathrm{x}$ \\
EQ-5D-5L & $\mathrm{x}$ & & $\mathrm{x}$ & & $\mathrm{x}$ & & $\mathrm{x}$ \\
PAM & $\mathrm{x}$ & & $\mathrm{x}$ & & $\mathrm{x}$ & & $\mathrm{x}$ \\
ICECAP-A & $\mathrm{x}$ & $\mathrm{x}$ & $\mathrm{x}$ & $\mathrm{x}$ & $\mathrm{x}$ & $\mathrm{x}$ & $\mathrm{x}$ \\
PCQ (adapted) & $\mathrm{x}$ & $\mathrm{x}$ & $\mathrm{x}$ & $\mathrm{x}$ & $\mathrm{x}$ & $\mathrm{x}$ & $\mathrm{x}$ \\
\hline MCQ (adapted) & $\mathrm{C}$ & & &
\end{tabular}

EQ-5D-5L, EuroQol-5D-5L; ICECAP-A, ICEpop CAPability measure for Adults; MCQ, Medical Consumption Questionnaire; PACIC, Patient Assessment of Chronic Illness Care; PAM, Patient Activation Measure; PCQ, Productivity Cost Questionnaire.

an instrument to measure outcomes. Outcomes will be measured seven times, including a baseline measurement before any experience in working with the ABCC tool. The follow-up measurements will take place at 3 , $6,9,12,15$ and 18 months after the first consultation with the healthcare provider. Table 1 provides a list of the instruments that will be used to measure health and cost outcomes. The PACIC, a valid and reliable questionnaire, measures perceived quality of care, and consists of 20 items. ${ }^{121319}$ Answers range from 1 (almost never) to 5 (almost always). Average scores will be calculated. Higher scores mean more frequent presence of the aspect of structured chronic care. The PACIC has been used in previous studies related to shared decision making and self-management. ${ }^{82021}$ The EuroQol-5D-5L (EQ-5D-5L) is a generic instrument for valuing health using five levels of severity in five dimensions of health, and also includes a Visual Analogue Scale (VAS) ${ }^{22}$ Answers from the descriptive system range from 1 (no problems) to 5 (severe problems). These will be converted into a single index value, using Dutch values. ${ }^{23}{ }^{24}$ The VAS ranges from 0 (worst imaginable health) to 100 (best imaginable health). The EQ-5D-5L is a valid and reliable questionnaire, and the most commonly used questionnaire to value health outcomes. ${ }^{25-30}$ The Patient Activation Measure (PAM) measures patients' activation, defined as the knowledge, skills, beliefs and behaviours that patients need to manage a chronic condition. ${ }^{31}$ The questionnaire consists of 13 questions on a 5-point scale. Scores range from 0 (lowest possible activation) to 100 (highest possible activation). The PAM shows good psychometric properties. ${ }^{32}{ }^{33}$ The ICEpop CAPability measure for Adults (ICECAP-A) is a valid questionnaire that measures capability well-being, defined as individuals' abilities to 'be' and 'do' the things that are important in life. ${ }^{34} 35$ The questionnaire consists of five questions. Answer options range from 1 (no capability) to 4 (full capability). A tariff value for an overall state will be determined based on values in the $\mathrm{UK}^{36}$ To measure costs related to medical consumption and productivity losses, the Medical Consumption
Questionnaire (MCQ) and the Productivity Cost Questionnaire (PCQ) were used to construct an appropriate questionnaire for this study. ${ }^{37}$ These questionnaires are carefully developed, are often used and are recommended in Dutch guidelines for economic evaluation of health-related interventions. ${ }^{38}{ }^{39}$ The adapted MCQ includes 16 questions related to the amount of contacts with healthcare providers. ${ }^{37}$ The adapted PCQ includes nine questions related to the impact of disease on the ability of a person to perform work. ${ }^{38}$ The items will be valued using standard cost prices in the Netherlands. ${ }^{40}$ Costs will be calculated by multiplying the volumes by cost prices per unit.

Questionnaires to measure the effects of the ABCC tool will be sent via email or by post to patients' homes if participants have no email. Online questionnaires will be completed via the Qualtrics XM platform. Healthcare providers will be asked to complete a short questionnaire about the general practice at baseline, which will be sent by post. Data will be stored at the Maastricht University Centre for Data and Information Management.

\section{Outcomes}

The primary outcome is change in perceived quality of care, as measured by the PACIC, compared with usual care after 18 months for the total group.

Secondary outcomes include:

1. Change in perceived quality of care, as measured by the PACIC, compared with usual care after 18 months for each condition separately.

2. Change in perceived quality of care, as measured by the PACIC, compared with usual care after 6 and 12 months for the total group and for each condition separately.

3. Change in generic health-related quality of life, as measured by the EQ-5D-5L, compared with usual care after 6,12 and 18 months for the total group and for each condition separately.

4. Change in patients' activation, as measured by the PAM, compared with usual care after 6,12 and 18 months for the total group and for each condition separately.

5. Change in capability well-being, as measured by the ICECAP-A, compared with usual care after 6, 12 and 18 months for the total group and for each condition separately.

6. Cost-effectiveness of the ABCC tool, from a societal perspective, compared with usual care after 18 months using the adapted PCQ and MCQ for costs and the EQ5D-5L and the ICECAP-A as outcomes and over lifetime, using decision analytical modelling.

\section{Statistical analyses}

Data will be analysed according to the intention-to-treat principle. Multiple imputation will be used to correct for missing data. First, the analyses will be conducted for the entire group (ie, patients with COPD, asthma, type 2 diabetes and heart failure). This allows for the inclusion 
of patients with multimorbidity. Second, the effects of the ABCC tool for patients with COPD, asthma, type 2 diabetes and heart failure will be analysed separately, regardless whether or not they have comorbid conditions. Because of the clustered design of the study, a multilevel analysis will be conducted with three levels: general practice, patient and measurement. Outcomes will be analysed using linear mixed models to account for the nesting of measurements within patients who are clustered in a general practice. The variables 'treatment arm', 'time' (categorical) and 'treatment arm by time interaction' will be included as fixed factors. Because it is not possible to randomise participants to the intervention or control group, factors that are hypothesised or known to be associated with perceived quality of care and/or the use of the ABCC tool will be considered as potential confounders. The allocation to the intervention or the control group depends on the access to the ABCC tool (ie, the availability of the ABCC tool in the electronic medical record system of the healthcare provider), and therefore the system that the general practice is using. These general practices might be located in different neighbourhoods, and consequently have another population composition. Therefore, potential confounders include: national background (native/Western foreigner/non-Western foreigner); multimorbidity (yes/no); educational level (low/middle/high); age (years; continuous); sex (man/ woman); body mass index ( $\mathrm{kg} / \mathrm{m}^{2}$; continuous); smoking status (never/former/current); location of the general practice (urban/rural); general practitioner with specialisation in COPD, asthma, type 2 diabetes or heart failure (yes/no); year of graduation managing general practitioner $(<10$ years ago/ $>10$ years ago); general practice in a health centre that is, availability of pharmacy, psychologist or physiotherapist in same building (yes/no); and the possibility of consulting a specialist within primary care (yes/no). Each potential confounder will be separately added to the above-mentioned linear mixed model, and will be considered as a confounder if significantly associated with the outcome $(\mathrm{p} \leq 0.05)$. The unadjusted and adjusted treatment effects will be reported together with the corresponding $95 \%$ CIs and $p$ values. A $p$ value of $\leq 0.05$ will be considered statistically significant. As a sensitivity analysis, an inverse probability of treatment weighting using propensity scores will be applied to account for multiple potential confounders.

\section{Cost-effectiveness}

Cost-effectiveness will be evaluated in the short term and long term. The short-term analysis will adopt the time horizon of the data collection in the quasi-experiment study, which is 18 months. The long-term horizon will adopt patients' lifetime. Costs and effects will be assessed from a societal perspective, including healthcare, productivity and travel costs. An incremental cost-effectiveness ratio will be used, which is measured as the incremental cost per quality adjusted life years (QALYs) gained from baseline to follow-up at 18 months using EQ-5D-5L scores. ${ }^{24}$ Because the ABCC tool is likely to yield benefits beyond those captured in generic health-related quality of life and mortality, the ICECAP-A will be used to estimate a capability well-being adjusted life year in addition. ${ }^{34}$ Unit prices will be derived from the Dutch Guidelines on Health Economic Evaluation. ${ }^{40}$ For the short-term analysis, bootstrapping will be used to obtain CIs. To conduct the long-term analyses, decision analytical modelling will be used. Outcomes will be QALYs and/or life years and costs. Uncertainty will be further assessed in sensitivity and scenario analyses.

\section{Blinding}

Blinding of healthcare providers and patients is not possible due to the nature of the intervention, but the study team will be blind to the treatment arms in the dataset during data cleaning, handling of missing data, statistical analyses and drawing of conclusions.

\section{Sample size}

The primary outcome in the study will be measured using the PACIC. Regarding the PACIC, little is known about the minimal important difference and SD in the Dutch population. A medium effect size of 0.51 was estimated based on the study of Slok $e t a l .{ }^{9}$ In this study, a difference in means between the intervention and control groups of 0.49 was found, with a pooled within-group SD of 0.96 . Based on an independent-samples t-test, a significance level $\alpha$ of 0.05 for two-sided testing, a power $(1-\beta)$ of $90 \%$ and an intervention: control ratio of 1:1, 82 patients need to be included per arm. As the patients are nested within general practices, we need to correct for this design effect (multiply by $1+(\mathrm{m}-1) * \mathrm{ICC} /$ intraclass correlation), where we assume an ICC of 0.05 and a mean number of patients per general practice $(\mathrm{m})$ of $10 .^{9}$ If corrected for the cluster design (multiplied by 1.45), unequal cluster sizes (divided by 0.9$)^{41}$ and dropout of $25 \%$ (divided by 0.75), 177 participants per arm are required. Assuming the availability of 10 patients per general practice, 18 general practices need to be included per arm. A total of 180 participants per arm (total 360 participants) from 18 general practices (total 36 general practices) will be included in the clustered quasi-experimental study. It is aimed to reach an equal distribution among the various chronic conditions. Unfortunately, this cannot be guaranteed because healthcare providers will not be instructed to aim for an equal distribution.

\section{Patient and public involvement}

Patients and healthcare providers were actively involved during the development of the ABCC tool. The study has an advisory group, including patients, patient organisations and healthcare providers.

\section{ETHICS AND DISSEMINATION}

Ethical approval was obtained from the Medical Ethics Committee Zuyderland-Zuyd Heerlen, the Netherlands 
(METCZ20180131). Written or electronic informed consent will be obtained from all study participants (online supplemental file 1). Study results will be published in international peer-reviewed journals, and will be presented at national and international conferences. The study started in November 2019 and is anticipated to continue until November 2021. On completion of the trial, data requests can be submitted to the corresponding author.

\section{DISCUSSION}

This quasi-experimental study assesses the effectiveness and cost-effectiveness of the ABCC tool. The ABCC tool aims to assess and visualise the burden of disease, to encourage shared decision making, to stimulate selfmanagement and to formulate care plans with personalised goals. The ABCC tool will be provided at the level of general practice. A total of 36 general practices and 360 patients will be included in the study, of which 18 general practices and 180 patients will be instructed to use the ABCC tool during usual consultation. The primary outcome of the study is the change in the patient's perception of quality of care. The follow-up time is 18 months.

People with chronic conditions have a need for holistic and continuing care, for shared decision making, and for help with self-management. ${ }^{42}$ Patient-centred care is growing in prominence. Studies have shown that patientcentred care leads to higher levels of adoption of healthy behaviour, increased adherence, better health outcomes, better care experiences and decreased healthcare utilisation. ${ }^{343}$ However, other studies show that the evidence in primary care is ambiguous. ${ }^{445}$ Because the current study builds on a robust evidence base surrounding the effectiveness of the $\mathrm{ABC}$ tool, we hypothesise that the $\mathrm{ABCC}$ tool significantly improves perceived quality of care and quality of life, and that it is cost-effective.

A strength of the current study is its pragmatic approach, including broad inclusion criteria and a wide range of outcomes including patient experience of care, quality of life and resource utilisation. The pragmatic approach reflects its effectiveness in real-world implementation and therefore enhances external validity. Because it is not feasible to randomise, the risk of confounding due to an unequal distribution of prognostic factors might be increased. However, we do not expect major differences between the intervention and control groups, because the allocation is only based on the information system that a general practice is using. Factors that are hypothesised or known to be associated with the intervention and the outcome will be considered as potential confounders. Another limitation is that most outcomes in the study are subjective. Furthermore, the PACICis used only to a limited extend in the Netherlands in settings that resemble our study. Therefore we estimated the effect size on a single study. The assumed effect size in our study may therefore result in an underestimation or overestimation of the power. Furthermore, the blinding of healthcare providers is not possible. This can lead to selection bias, because the healthcare providers are recruiting patients. Because a healthcare provider in the control group knows that the intervention will not be used, patients with different characteristics might be invited for the study compared with patients that are invited by healthcare providers in the intervention group. In this latter group, patients that are more easily motivated to work on their health might be more likely to be invited. Regarding the generalisability of the results, another limitation might be that the study is only conducted in primary care, and therefore no conclusion can be drawn regarding its effectiveness in other settings, such as secondary or tertiary care.

Concomitantly to this study, a context evaluation and process evaluation will be conducted among healthcare providers to better understand the use of the ABCC tool. Besides, a qualitative study will be conducted among patients to understand the use and satisfaction of the ABCC tool. The protocol for the context evaluation and process evaluation will be published separately.

The ABCC tool is aimed to improve patients' experience of individualised patient-centred care and to improve quality of life. Besides, the self-management that the ABCC tool aims for, might reduce healthcare costs, and therefore might contribute to sustainable healthcare. If the ABCC tool proves to be effective and cost-effective in primary care, the study has significant public health importance, as it can facilitate implementation and adoption of the intervention among primary care.

\section{Author affiliations \\ ${ }^{1}$ Department of Family Medicine, Care and Public Health Research Institute (CAPHRI), Maastricht University, Maastricht, The Netherlands \\ 2Department of Clinical Epidemiology and Medical Technology Assessment (KEMTA), Maastricht University Medical Centre, Maastricht, The Netherlands \\ ${ }^{3}$ Department of Methodology and Statistics, Care and Public Health Research Institute (CAPHRI), Maastricht University, Maastricht, The Netherlands}

Acknowledgements We would like to thank Mascha Twellaar and Silvia Bours for their assistance during the preparations of the study.

Contributors EAB, DC, AHMG-S and OCPvS designed the study. EAB drafted the manuscript. BW was consulted in writing about statistics, and MJ helped with writing about cost-effectiveness. The paper was critically revised by DC, MJ, LCEMK, OCPVS, BW and AHMG-S. All authors critically reviewed and revised the final version of the manuscript. All authors have read and approved the final manuscript.

Funding This study was funded by the Netherlands Organisation for Health Research and Development (104006001).

Disclaimer The funders in the study had no role in the study design; nor will they have a role in data collection, analysis, interpretation of the data or writing of the report.

Competing interests None declared.

Patient consent for publication Not required.

Provenance and peer review Not commissioned; externally peer reviewed.

Supplemental material This content has been supplied by the author(s). It has not been vetted by BMJ Publishing Group Limited (BMJ) and may not have been peer-reviewed. Any opinions or recommendations discussed are solely those of the author(s) and are not endorsed by BMJ. BMJ disclaims all liability and responsibility arising from any reliance placed on the content. Where the content includes any translated material, BMJ does not warrant the accuracy and reliability of the translations (including but not limited to local regulations, clinical guidelines, 
terminology, drug names and drug dosages), and is not responsible for any error and/or omissions arising from translation and adaptation or otherwise.

Open access This is an open access article distributed in accordance with the Creative Commons Attribution Non Commercial (CC BY-NC 4.0) license, which permits others to distribute, remix, adapt, build upon this work non-commercially, and license their derivative works on different terms, provided the original work is properly cited, appropriate credit is given, any changes made indicated, and the use is non-commercial. See: http://creativecommons.org/licenses/by-nc/4.0/.

ORCID iD

Esther A. Boudewijns http://orcid.org/0000-0001-9087-1712

\section{REFERENCES}

1 Salisbury C, Man M-S, Bower P, et al. Management of multimorbidity using a patient-centred care model: a pragmatic cluster-randomised trial of the 3D approach. Lancet 2018;392:41-50.

2 Ory MG, Ahn S, Jiang L, et al. Successes of a national study of the chronic disease self-management program: meeting the triple AIM of health care reform. Med Care 2013;51:992-8.

3 Eaton S, Roberts S, Turner B. Delivering person centred care in long term conditions. BMJ 2015;350:h181.

4 Stewart M, Brown JB, Weston W, et al. Patient-Centered medicine: transforming the clinical method. CRC press, 2013.

5 Bodenheimer T, Wagner EH, Grumbach K. Improving primary care for patients with chronic illness. JAMA 2002;288:1775-9.

6 Little P, Everitt $\mathrm{H}$, Williamson I, et al. Preferences of patients for patient centred approach to consultation in primary care: observational study. BMJ 2001;322:468.

7 Slok AHM, in 't Veen JCCM, Chavannes NH, et al. Development of the assessment of burden of COPD tool: an integrated tool to measure the burden of COPD. NPJ Prim Care Respir Med 2014:24:1-4.

8 Slok AHM, Bemelmans TCH, Kotz D, et al. The assessment of burden of COPD (ABC) scale: a reliable and valid questionnaire. COPD 2016:13:431-8.

9 Slok AHM, Kotz D, van Breukelen G, et al. Effectiveness of the assessment of burden of COPD (ABC) tool on health-related quality of life in patients with COPD: a cluster randomised controlled trial in primary and hospital care. BMJ Open 2016;6:e011519.

10 Slok AHM, Twellaar M, Jutbo L, et al. 'To use or not to use': a qualitative study to evaluate experiences of healthcare providers and patients with the assessment of burden of COPD (ABC) tool. npj Prim Care Resp Med 2016;26:1-8.

11 Boudewijns EA, Claessens D, van Schayck OCP, et al. ABC-tool reinvented: development of a disease-specific 'Assessment of Burden of Chronic Conditions (ABCC)-tool' for multiple chronic conditions. BMC Fam Pract 2020;21:1-7.

12 Glasgow RE, Wagner EH, Schaefer J, et al. Development and validation of the patient assessment of chronic illness care (PACIC). Med Care 2005;43:436-44.

13 Wensing M, van Lieshout J, Jung HP, et al. The patients assessment chronic illness care (PACIC) questionnaire in the Netherlands: a validation study in rural general practice. BMC Health Serv Res 2008;8:182.

14 Makoul G, Clayman ML. An integrative model of shared decision making in medical encounters. Patient Educ Couns 2006;60:301-12.

15 Hoes A, Voors A, Rutten F, et al. NHG-standaard Hartfalen (tweede herziening). Huisarts Wet 2010;53:368-89.

16 Snoeck-Stroband J, Schermer T, Van Schayck C, et al. NHGstandaard COPD (derde herziening). Huisarts Wet 2015;58:198-211.

17 Smeele I, Barnhoorn MJM, Broekhuizen BDL, et al. NHGstandaard astma bij volwassenen (derde herziening). Huisarts Wet 2015;58:142-54

18 Rutten G, De Grauw W, Nijpels G, et al. NHG-standaard diabetes mellitus type 2 (derde herziening). Huisarts Wet 2013;56:512-25.

19 Aung E, Ostini R, Dower J, et al. Patient assessment of chronic illness care (PACIC) in type 2 diabetes: a longitudinal study. Eval Health Prof 2016;39:185-203.

20 Vermunt NPCA, Harmsen M, Westert GP, et al. Collaborative goal setting with elderly patients with chronic disease or multimorbidity: a systematic review. BMC Geriatr 2017;17:167.
21 Schillinger D, Handley M, Wang F, et al. Effects of self-management support on structure, process, and outcomes among vulnerable patients with diabetes: a three-arm practical clinical trial. Diabetes Care 2009;32:559-66.

22 Herdman M, Gudex C, Lloyd A, et al. Development and preliminary testing of the new five-level version of EQ-5D (EQ-5D-5L). Qual Life Res 2011:20:1727-36.

23 van Hout B, Janssen MF, Feng Y-S, et al. Interim scoring for the EQ$5 \mathrm{D}-5 \mathrm{~L}$ : mapping the EQ-5D-5L to EQ-5D-3L value sets. Value Health 2012;15:708-15.

24 Versteegh M, Vermeulen K, Evers S, et al. Dutch tariff for the fivelevel version of EQ-5D. Value Health 2016;19:343-52.

25 Bae E, Choi S-E, Lee H, et al. Validity of EQ-5D utility index and minimal clinically important difference estimation among patients with chronic obstructive pulmonary disease. BMC Pulm Med 2020;20:73

26 Sullivan PW, Ghushchyan VH. EQ-5D scores for diabetes-related comorbidities. Value Health 2016;19:1002-8.

27 Crossman-Barnes C-J, Sach T, Wilson A, et al. The construct validity and responsiveness of the EQ-5D-5L, AQL-5D and a bespoke TTO in acute asthmatics. Qual Life Res 2020;29:619-27.

28 Hernandez G, Garin O, Dima AL, et al. EuroQol (EQ-5D-5L) validity in assessing the quality of life in adults with asthma: cross-sectional study. J Med Internet Res 2019;21:e10178.

29 Janssen MF, Lubetkin El, Sekhobo JP, et al. The use of the EQ$5 \mathrm{D}$ preference-based health status measure in adults with type 2 diabetes mellitus. Diabet Med 2011;28:395-413.

30 Nolan CM, Longworth L, Lord J, et al. The EQ-5D-5L health status questionnaire in COPD: validity, responsiveness and minimum important difference. Thorax 2016;71:493-500.

31 Hibbard JH, Mahoney ER, Stockard J, et al. Development and testing of a short form of the patient activation measure. Health Serv Res 2005;40:1918-30.

32 Skolasky RL, Green AF, Scharfstein D, et al. Psychometric properties of the patient activation measure among multimorbid older adults. Health Serv Res 2011;46:457-78.

33 Rademakers J, Nijman J, van der Hoek L, et al. Measuring patient activation in the Netherlands: translation and validation of the American short form patient activation measure (PAM13). BMC Public Health 2012;12:577.

34 Al-Janabi H, Flynn TN, Coast J. Development of a self-report measure of capability wellbeing for adults: the ICECAP-A. Qual Life Res 2012;21:167-76.

35 Keeley $\mathrm{T}$, Al-Janabi $\mathrm{H}$, Lorgelly $\mathrm{P}$, et al. A qualitative assessment of the content validity of the ICECAP-A and EQ-5D-5L and their appropriateness for use in health research. PLOS One 2013;8:e85287.

36 Flynn TN, Huynh E, Peters TJ, et al. Scoring the ICECAP-A capability instrument. Estimation of a UK general population tariff. Health Econ 2015;24:258-69.

37 Bouwmans C, Hakkaart-van Roijen L, Koopmanschap M. Handleiding iMTA medical cost questionnaire (iMCQ). Rotterdam: iMTA, Erasmus Universiteit Rotterdam, 2013.

38 Bouwmans $\mathrm{C}, \mathrm{Krol} \mathrm{M}$, Severens $\mathrm{H}$, et al. The iMTA productivity cost questionnaire: a standardized instrument for measuring and valuing health-related productivity losses. Value Health 2015;18:753-8.

39 Goossens ME, Rutten-van Mölken MP, Vlaeyen JW, et al. The cost diary: a method to measure direct and indirect costs in costeffectiveness research. J Clin Epidemiol 2000;53:688-95.

40 Nederland Z. Richtlijn voor Het uitvoeren van economische evaluaties in de gezondheidszorg. Diemen: Zorginstituut Nederland, 2016.

41 van Breukelen GJP, Candel MJJM, Berger MPF. Relative efficiency of unequal versus equal cluster sizes in cluster randomized and multicentre trials. Stat Med 2007;26:2589-603.

42 Mirzaei M, Aspin C, Essue B, et al. A patient-centred approach to health service delivery: improving health outcomes for people with chronic illness. BMC Health Serv Res 2013;13:251.

43 Fix GM, VanDeusen Lukas C, Bolton RE, et al. Patient-centred care is a way of doing things: how healthcare employees conceptualize patient-centred care. Health Expect 2018;21:300-7.

44 Mead N, Bower P. Patient-centredness: a conceptual framework and review of the empirical literature. Soc Sci Med 2000;51:1087-110.

45 Michie S, Miles J, Weinman J. Patient-centredness in chronic illness: what is it and does it matter? Patient Educ Couns 2003;51:197-206. 